\title{
LA GEOLINGÜÍSTICA: CONSIDERACIONES SOBRE LA DIMENSIÓN ESPACIAL DEL LENGUAJE
}

\author{
JUAN MANUEL HERNÁNDEZ CAMPOY \\ (Universidad de Murcia)
}

\begin{abstract}
Until very recently, the concepts of space and spatiality and their, on the other hand, necessary integration in the social theory have played a role as scarcely emphasized as marginal in the evolution of the sociological thought. Time, in terms of historical developments, has always been considered as the principle contextual unit of social theory, while space, on the contrary, has been a secondary dimension. Linguistics has also taken part in this historicism that has excluded spatial aspects from its both dialectological and sociolinguistic studies. It has been with the advent of the New Human Geography arisen from the quantitative revolution when space and spaciality have become relevant not only in Social and Human Sciences in general but also in Linguistics in particular. The aim of this paper is to show and analyze the consequences of this interdisciplinary revolution which has resulted in a new discipline called Geolinguistics conceived as the confluence of Dialectology, Sociolinguistics and Human Geography.
\end{abstract}

\section{Introducción: el Espacio y la Espacialidad en las Ciencias Sociales y Humanas}

Hasta muy recientemente, los conceptos de espacio y espacialidad y su, por otra parte, necesaria, integración en la teoría social han jugado un papel tan poco destacado como marginal en el desarrollo del pensamiento sociológico. En efecto, el tiempo, traducido en desarrollos históricos, siempre se ha considerado la unidad contextual principal de la teoría social, mientras que, por el contrario, al espacio se le ha relegado a una posición ciertamente secundaria. Esta predilección por los aspectos temporales más que por los espaciales ha sido criticada de forma 
manifiesta por numerosos geógrafos y sociólogos. David Britain (1991: 195-197) destaca algunas reivindicaciones en este sentido:

Space was treated as the dead, the fixed, the undialectical, the immobile. Time on the contrary was richness, fecundity, life, dialectict ...

Michel Foucault (1980: 70)

Space is not an empty dimension along which social groupings become structured, but has to be considered in terms of its involvement in the construction of systems of interaction.

Anthony Giddens (1984: 368)

An essentially historical epistemology continues to pervade the critical consciousness of modern social theory. It still comprehends the world primarily through the dynamics arising from the emplacement of social being and becoming in the interpretative contexts of time [...] This historicism [...] has tended to occlude a comparable critical sensibility to the spaciality of social life, a practical theoretical consciousness that sees the life-world of being creatively located not only in the making of history, but also in the construction of human geographies, the social production of space and the restless formation and reformation of geographical landscapes.

Edward Soja (1989: 10-11)

El espacio, para David Britain (1991: 199-200), puede ser de tres tipos: el euclidiano, el social y el perceptible. El primero es el espacio puramente físico de la naturaleza; esto es, el espacio objetivo, geométrico y completamente asocial de las matemáticas y la física. El segundo, por el contrario, es el espacio creado por la acción del hombre y la organización social mediante la manipulación del paisaje. El tercero, el espacio perceptible, es el entorno inmediato, y no tan inmediato, percibido por la gente en la vida cotidiana, el cual incide muy decisivamente en su comportamiento y las actitudes generadas. La confluencia de los tres constituye la espacialidad, que siempre se encuentra, según Allan Pred (1985: 338), en estado de transformación, siendo constantemente modelada y remodelada por la acción de la sociedad. En una ciencia social, además, ninguno de estos tres tipos deben ser tratados por separado, dado que, durante la producción de la espacialidad, el espacio geométrico puede ser adoptado y hecho social, pero este último no puede concebirse independientemente del primero: «... social space can never be entirely free of, say, the physical friction of distance» (David Britain 1991: 200); y, del mismo modo, nuestras percepciones y sistemas de valores asociados a nuestro entorno, aunque influidos por los espacios euclidiano y social, pueden también incidir en el modo de adopción del espacio a través de los sentidos. 
Todo elemento histórico (temporal) de los hechos humanos, pues, ha sido un rasgo esencial y común a las diferentes tendencias de la ciencia social hasta la mitad del presente siglo aproximadamente. La historia se elevó a categoría explicativa de la realidad social al considerar que esta última era resultado de un desarrollo eminentemente temporal y, consiguientemente, la descripción de dicho desarrollo desde su origen era esencial e indispensable para entender la realidad. Para Wilhelm Dilthey (1883: 82), toda acción humana llevaba en sí la historia, era fruto del devenir y además estaba sometida al cambio. Por ello, concebían, él y sus contemporáneos, que la realidad social era siempre histórica y que histórico era necesariamente todo objeto de las ciencias humanas. De este modo, estas, las ciencias humanas, creadas históricamente, no hacían más que dar cuenta de esa historicidad esencial de la realidad. Tradicionalmente, la Geografía y la Historia, por ejemplo, siempre se han atribuido un excepcionalismo que las caracterizaba como ciencias singulares entre las demás y que las excusaba frente a las revoluciones científicas; así, la tradición historicista consideraba que ambas se enfrentan con explicar lo único siempre a través del tiempo.

Tampoco estuvieron al margen de estas concepciones historicistas la Lingüística y la Dialectología Tradicional, ambas complentamente desvinculadas entonces. Tanto para la Linguística Histórica, con su teoría de las ondas, como para la Filología Comparada, con su teoría del árbol genealógico, el tiempo y los desarrollos históricos conformaban sus modelos de análisis, como lo reflejan sus estudios de naturaleza eminentemente diacrónica. En este contexto de la lingüística histórica y comparada, la Dialectología Tradicional no está exenta de esta tendencia historicista, a pesar de ocuparse del estudio del variacionismo presente en el lenguaje desde una perspectiva geográfica. La diferencia en su tratamiento estriba en el enfoque, coincidiendo con el de la Geografía Humana de aquellos momentos anteriores a 1960, que se ocupaba de 'las regiones', con 'la diferencia' y 'la distintividad' como rasgos más sobresalientes de su objeto de estudio. Esto es, en lugar de centrarse en los procesos o estructuras espaciales, analizan las áreas de modo individual, buscando sus características únicas, y con total independencia de las circundantes. Del mismo modo, los estudios dialectológicos, impulsados por la hipótesis de los neogramáticos y asumiendo que los dialectos eran entidades discretas, homogéneas y separadas por fronteras dialectales, se dedicaban a trazar isoglosas en mapas lingüísticos para 'diseccionar' y representar esa discontinuidad de las áreas dialectales. Los dialectólogos tradicionales ni tuvieron en cuenta jamás una dimensión social ni le dieron al espacio más importancia que la de considerarlo un mero continente de variación linguística. Su percepción del espacio, por tanto, era más bien de modo estático. Al limitarse al tiempo y la historicidad para reconstruir la variación dialectal existente en el pasado, o presente, de una lengua, ignoraban el dinamismo de la sociedad, no evaluaban las posibles interrelaciones de los distintos lugares, y la función del espacio se reducía a la representación en 
mapas de la distribución geográfica de su variación fonético-fonológica, morfosintáctica o léxico-semántica: «The essential question of why two locations shared or did not share certain linguistic characteristics was not on the agenda» (David Britain 1991: 203).

La Sociolingüística también ha participado de este historicismo que ha marginado los aspectos del espacio físico en sus estudios. A pesar del empirismo científico que impulsó el desarrollo de esta disciplina y que destaca lo regular, lo general y lo neutro por encima de lo específico, lo único y lo individual, la función del tiempo y el desarrollo histórico se ha mantenido constante, aunque se ha incorporado el espacio social (lo social) y el ámbito urbano en sus análisis sobre la heterogeneidad del lenguaje. Los sociolingüistas ponen un gran énfasis en el urbanismo y su especificidad (Soja 1989: 69), puesto que la investigación en la ciudad se considera la forma más adecuada de tener acceso a las comunidades más fluidas y heterogéneas posibles. Sus estudios del cambio lingüístico los realizan desde una dimensión social recorriendo el espacio social (vertical) que determinan las clases sociales de un núcleo urbano aislado y su posible correlación con la variación lingüística, pero no atienden al espacio físico (horizontal) y su posible interrelación con el comportamiento de otros núcleos de población. Además de insistir en la organización espacial de la sociedad (clases), resulta necesario tener en cuenta aquellos aspectos relativos a la organización social del espacio. Si, como defiende Edward Soja (1989: 12), la sociedad es el resultado de la confluencia del tiempo, el espacio y el ser humano, es de desear, pues, que la espacialidad, en tanto que dimensión integrada por los espacios euclidianos, sociales y perceptibles, juegue un papel más relevante en el progreso que hace la investigación sociolingüística en la evidenciación de las interrelaciones existentes entre el lenguaje y la sociedad:

... only when we combine a critical understanding of the socio-spatial as well as linguistic ingredients in any situation of dialect contact can we arrive at something approaching a full appreciation of the sociolinguistic facts.

David Britain (1991: 258)

En el caso concreto de la difusión tanto social como geográfica de las innovaciones linguísticas, el análisis de la espacialidad a través del paradigma de lenguaje y geografía, o estudio del lenguaje en su contexto geográfico, también es crucial y, como Andrew Sayer (1985: 50) afirma, «space makes a difference», a pesar de que, hasta ahora ésta había sido desestimada:

An analysis of spatiality is critically important if we wish to fully understand the processes involved both in the diffusion of linguistic innovations and the development of typological differences in languages and dialects alike.

David Britain (1991: 251-252) 
El reciente impulso dado a la función del espacio y la espacialidad ha venido dado de la nueva Geografía Humana. La crisis de las concepciones historicistas y el surgimiento de las corrientes neopositivistas, o «revolución cuantitativa», en la ciencia en general, que también afectó a la Lingüística, tuvo su repercusión en el pensamiento geográfico con la Nueva Geografía o Geografía Cuantitativa como resultado: «... es siempre el positivismo y la reacción antihistoricista la que inspira, en lo fundamental, el trabajo de la nueva geografía cuantitativa» (Horacio Capel Sáez 1981: 394). Si la década de los años cincuenta fue fructífera a la vez que determinante en el desarrollo metodológico de las Ciencias Sociales en general, también lo fue para la Geografía en particular. Sería Fred K. Schaefer (1953) quien originó la revolución cuantitativa y teórica en este campo y puso de manifiesto que la Geografía es una ciencia como las demás y debe, por consiguiente, adoptar el método científico, con su lenguaje altamente formalizado común a todas las ramas del saber que posibilite la transferencia de teorías y conceptos de una disciplina científica a otra. Esto la llevó a entrar en contacto con algunas de ellas tales como la Economía, la Antropología, la Sociología, o la Psicología, y a abandonar sus relaciones con la Historia, que la hacía eminentemente descriptiva. De este modo, se produciría una transformación en los cometidos teóricos del pensamiento geográfico, pasando de la mera descripción a la explicación y predicción de fenómenos. Con trabajos fundamentales como los de Fred Schaefer (1953) y posteriormente William Bunge (1962) se cuestionó el tradicional «excepcionalismo» atribuido a la Geografía, que la caracterizaba como ciencia singular entre las demás: la tradición historicista consideraba que tanto la Geografía como la Historia se enfrentan con el problema de explicar lo único, para lo que, evidentemente, no es posible formular una ley. La voluntad explícita de ciencia positiva de los 'nuevos geógrafos' trata de llegar a la explicación científica y la formulación de leyes generales que posibiliten la predicción de fenómenos: «si una teoría no puede predecir es que no ha descubierto la regla de la realidad (William Bunge 1962: 2). Se proponen, pues, desarrollar una geografía humana cuantificada que gire en torno al espacio euclidiano, estableciendo leyes, procesos y relaciones espaciales que puedan explicarse enteramente mediante factores espaciales. Asumiendo que las regularidades existentes en la naturaleza física se encuentran también en otros órdenes de la realidad, como la social, se plantean como objetivo primordial de la disciplina la búsqueda de leyes que gobiernan la distribución espacial de determinadas características sobre la superficie terrestre: «la ciencia no está tan interesada en los hechos individuales como en los patrones que presentan» ${ }^{\mathrm{I}}$. Insisten en el estudio de las relaciones entre los lugares más que en el estudio de los

\footnotetext{
${ }^{1}$ Fred K. Schaefer (1953), página 33 de la versión española; F.K. Schaefer (1971) Excepcionalismo en Geografía. Barcelona: Ediciones de la Universidad de Barcelona, traducción e introducción realizada por Horacio Capel Sáez (2ªdición, 1974).
} 
lugares en sí; esto es, se centran en la investigación no de qué fenómenos hay y dónde se dan, sino en las causas por las que aparecen allí y no en otro lugar. Así, se convierten en temas destacados de investigación cuestiones relativas al espacio terrestre: la organización espacial, las distribuciones y asociaciones espaciales, las estructuras espaciales, la localización espacial de la población y de las actividades, y todo tipo de regularidades espaciales y la covariación de los fenómenos ${ }^{2}$. Optan decididamente los 'nuevos geógrafos' por el desarrollo del conocimiento que será el de la formulación de hipótesis mediante el procedimiento hipotético-deductivo y contrastación mediante la observación y la experimentación. La matematización de la disciplina vendría sustentada por investigadores como David Harvey (1969), quien insistía en que, puesto que la matemática es el lenguaje de la ciencia, también debe serlo de la Geografía. Para expresar con precisión y claridad los resultados de la investigación científica y para evitar contradicciones, se autoexigirán el empleo del lenguaje matemático y de la lógica, siendo la verificabilidad un criterio fundamental, y se plantearán la causalidad en términos de probabilidad: modelos probabilísticos $^{3}$. Estos objetivo, la búsqueda de explicaciones empíricas de aquellas regularidades existentes en la naturaleza física, se han manifestado en las investigaciones de los geógrafos Torsten Hägestrand (1952, 1957, 1965a, 1965b, 1966, 1967a, 1967b, 1969, 1970), Allan Pred (1967, 1977, 1985), Derek Gregory (1975), Edward Soja (1989) o John Urry (1985), que ejercerán una gran influencia no sólo en su disciplina, sino también en otras, como más adelante veremos.

\footnotetext{
${ }^{2}$ La utilización de teorías físicas para explicar por analogía situaciones que se producen en la Geografia Humana vino dada a partir de las propuestas de John Q. Stewart y William Warnzt (véanse John Q. Stewart 1947 y John Q. Stewart \& William Warnzt 1958a y 1958b), quienes se esforzaron por establecer las relaciones funcionales entre la Física y las Ciencias Sociales desarrollando una física social de la población mediante análisis macroscópico. Es decir, proponían analizar las situaciones demográficas, económicas, políticas, y sociológicas empleando factores puramente físicos tales como tiempo, distancia, masa social, temperatura social, deseo y número de personas, etc. desde una perspectiva macroscópica. Este uso de teorías físicas en el pensamiento geográfico se convirtió en una práctica común entre los quantifiers, produciendo numerosos hallazgos que contribuían a consolidar las bases de la «nueva geografía» y a dar confianza entre los trabajadores de este campo. Así, se descubren la regla del rangotamaño, que establece una relación entre el tamaño de la ciudad mayor de un país y las restantes ciudades existentes en el mismo; la existencia de una regularidad en la disminución de las densidades de población a partir del centro de una metrópoli; leyes que rigen la distribución espacial y la jerarquía de los núcleos urbanos (teoría de los lugares centrales); las teorías de difusión de las innovaciones en el espacio y en el tiempo y los modelos de simulación; el uso de la teoría de grafos en el estudio de los distintos tipos de redes (transportes, fluviales); el concepto de ecosistema como modelo de la realidad, etc. Ejemplos de la utilización de teorías físicas a situaciones análogas producidas en Geografía, así como bibliografía oportuna pueden encontrarse en William Bunge (1962) y Peter Hagget (1965).

${ }^{3}$ William Bunge (1962: págs. 2-3) sostenía que las características de las teorías tenían que ser i) la claridad, que se consigue presentándolas de forma matemática, ii) simplicidad y generalidad, que implica minimizar las variables y aumentar la información, y iii) exactitud.
} 
El interés por el análisis de la dispersión geográfica de los elementos lingüísticos se ha desarrollado igualmente no sólo en el seno de la Lingüística sino también desde la Geografía Humana, que ha contemplado las actitudes sociales y los sistemas de redes intra e intercomunitarias en su intento por diseñar modelos dinámicos de difusión. La Dialectología Urbana, el estudio de los dialectos urbanos, ha pasado de ser un área de investigación completamente minoritaria en sus momentos iniciales a dominar las corrientes lingüísticas actuales. Paralelo a éste, y en concomitancia con su ímpetu original, ha sido el desarrollo de la Teoría de la Variación, con toda la serie de hipótesis y premisas que conlleva aceptar la variable como unidad estructural en el modelo gramatical. En estudios sociolingüísticos más recientes, pues, el espacio físico ha conseguido un mayor protagonismo y se ha puesto en el lugar que le corresponde para poder comprender la interrelación y dinamismo existentes entre el escenario social, el espacial y los procesos lingüísticos.

\section{La Geolingüística: Lenguaje y Geografía}

La Geolingüística es un área de estudio multidisciplinar y relativamente reciente que se ocupa de las relaciones existentes entre el lenguaje y la geografía. Supone el estudio espacial del lenguaje, o estudio del lenguaje en su contexto geográfico, además del social y cultural. Si la Dialectología Tradicional es eminentemente rural y la Sociolingüística Laboviana es eminentemente urbana, la Geolingüística es una línea de investigación interdisciplinar sobre las características espaciales del lenguaje y cuya naturaleza se gesta en la confluencia de tres áreas: la geografía linguíística (Dialectología Tradicional), la dialectología urbana (Sociolinguiística Laboviana) y la geografía humana (Geografía) (J.K. Chambers \& P.J. Trudgill 1980). Es decir, si considerar quién habla con quién, cuándo, cómo, qué y con qué fin resulta un planteamiento importante en la investigación sociolingüística, del mismo modo, considerar dónde se lleva a cabo esa operación desde un macronivel, dónde se localiza físicamente una comunidad lingüística, su interacción con otras y, a la vez, su interrelación con el comportamiento de otras comunidades en otros núcleos de población resulta de gran importancia para los estudios geolingüísticos.

La existencia de estos movimientos provocará, como afirma Jack Chambers (1982: 1), considerables avances en el futuro, además de evidenciar la constante y creciente actividad y solidez de los estudios de geolingüísticos: «Developments like these greatly enhance for a vital and theoretically salient subfield called geolinguistics». Sin embargo, la integración de estas tres diferentes líneas de trabajo en una llamada Geolingüística, que ofrezca una dimensión espacial dinámica y en la que son de gran importancia las interacciones espaciales entre las formas 
lingüísticas, ha sido en ocasiones considerada inmanejable e imposible. Así, lingüistas como Charles-James Bailey (1973: 86) consideran que la geografía constituye un factor irrelevante en los procesos de contacto y difusión de fenómenos lingüísticos por el carácter caótico de los mismos: «Geographical dispersions can be so chaotic as to challenge the plausibility of any hypotheses about the orderliness of language variation». La contestación de Jack Chambers a esta afirmación es lo suficientemente rotunda y clarificadora:

\begin{abstract}
Even prima facie, it is simply inconceivable that language, as a human properly, would not reflect the spatial relationships which bring human beings together and keep them apart. True, the reflection of language in geography may not be as simple as Bailey and others apparently expected, but that is surely no reason dismiss it altogether. What is required is a richer set of hypotheses about language variation and a more profound understanding of spatial networks and diffusion patterns. Jack Chambers (1982:2)
\end{abstract}

\title{
III. La Fundamentación Geolinguiística
}

Los principios que inspiran los estudios geolingüisticos coinciden plenamente con los de la Sociolingüística y la nueva Geografía Humana, puesto que éstas, al igual que el resto de las disciplinas científicas, se vieron afectadas por la crisis de las concepciones historicistas y el surgimiento de la corriente neopositivista, también conocida como la «revolución cuantitativa». Con la rigurosa adopción del método científico, la voluntad explícita de ciencia positiva de los geolingüistas trata de llegar a la explicación científica de los fenómenos linguísticos sobre el espacio y la formulación de leyes generales que posibiliten su predicción. Asumiendo que las regularidades existentes en la naturaleza física se encuentran también en otros órdenes de la realidad, se plantean como objetivo primordial la búsqueda de leyes que gobiernan la distribución espacial de determinadas características linguuísticas sobre la superficie terrestre. Esto les hace insistir menos en la investigación de qué fenómenos hay y dónde se dan, lo que sería meramente descriptivo en su propósito, y más en las causas por las que se dan allí y no en otro lugar, lo que es de carácter netamente explicativo y predictivo en su propósito. De este modo, se convierten en temas de especial interés para los geolingüistas cuestiones relativas a las características espaciales del lenguaje, en general, y de las lenguas, en particular: la organización espacial del lenguaje, la distribución e interacción de las lenguas, su estructura espacial, la localización espacial de las comunidades lingüísticas y su comportamiento, y todo tipo de regularidades linguiísticas sobre el espacio tanto físico como social y su posible covariación. El desarrollo del conocimiento será, por tanto, el de la formulación de hipótesis mediante el procedimiento hipotético- 
deductivo y contrastación mediante la observación y la experimentación. Igualmente, para expresar de modo rigurosamente preciso y claro los resultados de la investigación científica geolinguística, se autoexigirán el empleo del lenguaje matemático y de la lógica, siendo la verificabilidad un criterio fundamental y planteándose la causalidad en términos de probabilidad.

En este sentido, William Mackey (1988) critica la arbitrariedad de algunas disciplinas a la hora de establecer sus principios metodológicos de observación e interpretación en sus análisis y aboga por la uniformidad de criterios ${ }^{4}$. Él distingue cuatro estadios esenciales que, en su opinión, deberían ser estandarizados en todo proceso procedimental de investigación en Geolinguiistica: i) observación, ii) descripción, iii) interpretación y, finalmente, iv) predicción. A la hora de planificar un estudio geolinguiístico primeramente hay que decidir el objeto de la observación (una lengua, un dialecto, una forma lingüística, etc.). El siguiente paso consiste en describir la unidad observada siguiendo unos procesos de identificación, localización, segmentación territorial, función, y evolución de la misma. Teniendo a nuestra disposición la suficiente información descriptiva, podemos plantearnos toda una serie de cuestiones interpretativas desde la perspectiva geolingüística como la distribución de la unidad de análisis, su poder de atracción, potencial, estatus, y, en su caso, su expansión, extinción o reposición/sustitución. No con el ánimo de profetizar, puesto que esto no es posible en las Ciencias Sociales, según William Mackey (1988: 38), con los resultados obtenidos del análisis anterior puede llegarse a establecer predicciones sobre el futuro de las lenguas, dialectos o de determinadas formas lingüísticas (fonético-fonológicas, morfológicas, sintácticas o semánticas) sobre la base de su evolución pasada y de su estatus presente.

\footnotetext{
${ }^{4}$ «In order to benefit from the enormous potential of these disciplines - in data, method and techniqueit is necessary that some basic principles of observation and interpretación be applied uniformly to each of them, at least as their output concerns the language varieties, the language users and the language areas of the world» (William Mackey 1988: 44).
} 


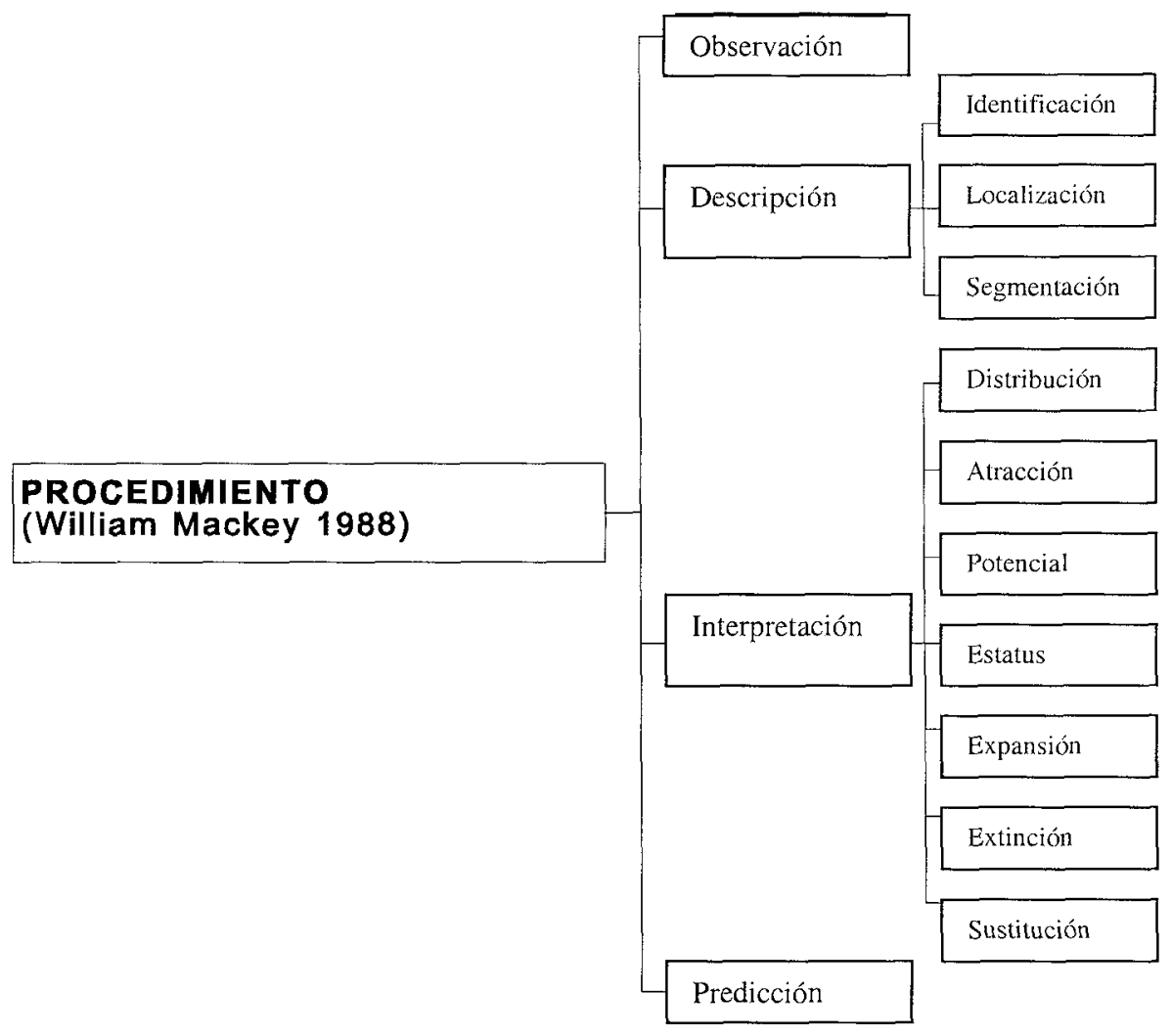

Atendiendo a la cobertura de este campo de estudio, al igual que ocurre en el espectro de lenguaje y sociedad (véanse Trudgill 1978 o Hernández Campoy 1993c), en el de lenguaje y geografía también se corre el riesgo de que las investigaciones se realicen con unos objetivos tendentes más a aspectos geográficos que lingüísticos y viceversa, por su propia naturaleza interdisciplinar y aun utilizando los mismos datos e incluso la misma metodología. De modo similar a como hizo Peter Trudgill (1978 y 1983) con respecto al paradigma sociolingüístico, nos atreveríamos a diferenciar en el geolinguístico dos tendencias principales: aquellos estudios que emplean datos de la Geografía Humana con propósitos tanto lingüísticos como geográficos y aquellos con fines puramente lingüísticos. La primera línea sería la liderada por Colin Williams junto con William Mackey, Harold Carter, Thomas Lundén, William Walter Bostock, J.E. Ambrose, Charles W.J. Withers, W.T.R. Pryce, Don Cartwright, Paul White, Humphrey Lloyd 
Humphreys, Kenneth MacKinnon, Clive James, John de Vries, John Edwards, muchos de ellos recogidos en Colin Williams (1988, 1991 y 1994), William Mackey (1983y 1988), Robert L. Cooper (1982 y 1989), Wilfried Stölting-Richert (1988), Robert L. Cooper \& Bernard Spolsky (1991), James W. Tollefson (1991), E.V. Gunnemark (1991), y las revistas Geolinguistics de la American Society for Geolinguistics y Working Papers in Geolinguistics de la Universidad de Staffordshire, principalmente. Se ocupa de cuestiones como el desarrollo social, espacial, cultural y político de las lenguas minoritarias y su trabajo coincide, en muy buena parte, pensamos, con el de la Sociología del Lenguaje, en lo que se refiere a la planificación lingüística, el bilingüismo, la diglosia, el cambio de código, la lealtad y la mortandad linguística, si bien esta Geolingüística persigue un mayor componente espacial, como Colin Williams reconoce:

Place was to become an important determinant of behaviour in establishing the rules of code switching, the identification of diglossic situations and the development of vernacular and dialect speech forms.

Colin Williams (1988: 5)

La adopción de presupuestos geográficos en las investigaciones sociolinguísticas nos permitiría demostrar la incidencia tan directa que tienen la distancia, la topografía o el entorno desarrollado en el análisis de la estructura social, en general, y de las lenguas, en particular. Con este espíritu, pretenden consolidar la perspectiva espacial en los estudios lingüísticos modernos:

Whilst one would not wish to argue that geolinguistic analysis has yet reached the theoretical realism found in other parts of Human Geography, notably the analysis of 'social relations and spatial structures' [...], it does nevertheless offer a more positive, practical appreciation of the role of space, territory and scale as useful additional elements to be emphasized in a more holistic sociolinguistic practice.

Colin Williams (1988: 6)

Con las cada vez más frecuentes migraciones interurbanas e internacionales y el consiguiente aumento del uso de los principios del pluralismo cultural para contrarrestar las tendencias asimilatorias, las lenguas en contacto son, en muy numerosas ocasiones, sinónimo de lenguas en conflicto, principalmente cuando las divisiones lingüísticas se correlacionan muy estrechamente con otras diferencias socioculturales como la religión, el estilo de vida o la afiliación política (J.E. Ambrose \& C.H. Williams 1991: 308). Es por ello que la naturaleza de esta línea geolinguística de objetivos geográficos, y lingüísticos, se sustenta sobre una base manifiesta y fundamentalmente interdisciplinar e intercultural: 
Underlying this approach was a conviction that the contemporary concerns with social planning, modernization and development were best served by recognicing their complexity and by the adoption of cross-cultural and inter-disciplinary perspectives.

Colin Williams (1988: ix)

La segunda línea geolingüística, la puramente lingüística, sería la liderada por Peter Trudgill (1974b, 1975b, 1986, 1989, 1990b y 1990c), y desarrollada con los trabajos de E. Afendras (1969, 1970a y 1970b), Gherard Hard (1972), Stanley Newman (1974), Robert Callary (1975), C. Elert (1976), Peter Trudgill \& Tina Foxcroft (1978), Arne Kjell Foldvik (1979), Marinel Gerritsen \& Frank Jansen (1980), Jack Chambers \& Peter Trudgill (1980), Lesley Milroy (1980), D. Larmouth (1981), Jack Chambers (1982), James Milroy \& Lesley Milroy (1985), Jack Chambers \& Margaret Hardwick (1986), Henning Andersen (1986), Penelope Eckert (1988), Karl Inge Sandred (1988), Rosina Lippi-Green (1989) David Britain (1991), Paul Kerswill (1991, 1994, 1995a y 1995b), Guy Bailey, Tom Wikle, Jan Tillery \& Lori Sand (1991 y 1993) o Walt Wolfram \& Natalie Schilling-Estes (1996). Si la línea de Colin Williams concibe esta disciplina como una nueva rama de la Geografia Humana y, por consiguiente, como un modo de hacer geografía, la línea trudgilliana la considera una rama dentro del paradigma de la Sociolinguiística y un modo de hacer linguística: «Its goal, like any other subdiscipline of modern linguistics, is to elucidate the most accesible system of human knowledge, the capacity for language» (Chambers \& Trudgill 1980: 206) ${ }^{5}$. La naturaleza de esta línea de estudios es también interdisciplinar desde su misma concepción, como Jack Chambers \& Peter Trudgill (1980: 206) han subrayado: «We conceive the confluence of these three streams - dialect geography, urban dialectology, and human geography- as a unified discipline». Combina, pues, la inmensa, y a la vez rica, cantidad de datos compilados en los atlas linguísticos por los dialectólogos, el rigor metodológico de la Sociolinguíística Laboviana y una mayor sensibilidad por los aspectos del espacio, como son las redes espaciales y la difusión de las innovaciones a través de las mismas, que viene dada de la Geografía. Su objeto de estudio es la difusión geográfica de las innovaciones lingüísticas dentro del marco de la teoría de la variación. Para ello, Trudgill (1983: 54) sostiene que los dialectólogos «no deberían contentarse simplemente con describir la distribución geográfica de los rasgos lingüísticos distintivos sino que también deberían ocuparse

\footnotetext{
${ }^{5}$ De hecho, Colin Williams (1991: 315) reconoce la mayor vinculación de su línea de cstudios geolinguísticos con la Sociología del Lenguaje y admite la mayor cobertura que ofrece la disciplina con otras líneas de investigación, como es la trudgilliana: «We are conscious that the largely underdeveloped ficld of geolinguistics and its parent discipline of Geography has much to offer co-workers in the Sociology of Language and Sociolinguistics. Our focus has been upon linguistic minorities in decline, but our approaches and methodologies are capable of wider application to most situations of language contact».
} 
de explicar esta distribución»; en otras palabras, diciendo exactamente por qué y cómo los rasgos lingǘsticos distintivos en proceso de cambio lingǘstico se difunden de un lugar o grupo social a otro. Así, podríamos entender con mayor precisión los mecanismos sociolingüísticos que subyacen a la distribución geográfica de las innovaciones linguísticas. De este modo, los dialectólogos tienen que emplear determinadas técnicas y conceptos teóricos desarrollados por los geógrafos sociales. Los mapas dialectales deberían ser mejorados considerablemente mediante el uso de las técnicas cartográficas de la Geografía, como las desarrolladas por el sueco Hägerstrand (1952), quien sostenía que «when studying changes we cannot draw boundary lines and observe their displacements. Instead we must ascertain the spatial diffusion of ratios»: se divide el terreno de manera uniforme en una serie de áreas, o casillas, y los investigadores tienen que calcular el porcentaje de uso de un determinado rasgo distintivo lingüístico en cada una de ellas, en determinados momentos del tiempo, del mismo modo que William Labov calculó los porcentajes de las casillas de diferentes clases sociales; esto es, tienen que mostrar no sólo la posición geográfica de un fenómeno específico sino incluso los factores realmente decisivos en estos procesos; nos referimos a la densidad de población y distribución social, la distancia, y el sistema lingüístico en sí como factor de resistencia con respecto a la posible semejanza lingüística preexistente. Conceptos teóricos procedentes de la Geografía Social, como modelos de difusión, efecto de vecindad y modelos de gravedad, pueden dar cuenta de la relativa densidad de población de áreas adyacentes, las redes de conexión entre un área determinada y grandes centros de población, la posición geográfica de una innovación, el grupo social innovador, el relativo prestigio de determinadas variedades, la distancia linguística entre las variedades, y el sistema linguíístico en sí como factor de resistencia. La aplicación de estas técnicas en los mapas y el conocimiento por parte de los dialectólogos de estos conceptos teóricos procedentes de la Geografia podrían hacer que la Lingiiústica averiguase la difusión geográfica de rasgos distintivos lingüísticos, o lo que es lo mismo, por qué las innovaciones lingüísticas surgen y se difunden a un núcleo $\mathrm{A}$ desde un núcleo $\mathrm{B}$ y no desde un núcleo $\mathrm{C}$. Por consiguiente, del mismo modo que la variable lingüística, con la ayuda de la teoría y métodos sociológicos, puede perfeccionar nuestro conocimiento de la relación existente entre el lenguaje y la sociedad, también la variable lingüística, junto con la serie de revelaciones metodológicas y teóricas procedentes de la Geografía Humana, puede perfeccionar nuestro conocimiento de las relaciones entre el lenguaje y la geografía, asî como el escenario geográfico del cambio lingüístico:

A dynamic dialectology or geolinguistics making use of time-incorporating geographical diffusion models and sociolinguistic and geographical techniques that permit the handling of gradient phenomena, may be better able to describe and even 
explain some of the processes involved in the geographical diffusion of linguistic innovations.

Peter Trudgill (1983: 87)

Las relaciones entre la Geolingüística y la Dialectología son claras. Los dialectólogos nunca consideraron su disciplina una ciencia social ni adoptaron sus principios teóricos y metodológicos hasta que apareció William Labov con la moderna Dialectología Urbana. La dimensión espacial tradicional tenía una naturaleza completamente estática, puesto que se limitaba a la representación cartográfica de la distribución geográfica de las formas linguísticas y no se planteaban razonar la distribución geográfica de los fenómenos lingüísticos: «why a certain linguistic variety occurred in some part of the country and not in another» (Marinel Gerritsen 1988: 1575). De hecho, investigadores como Charles-James Bailey (1973: 86) consideran, como anteriormente hemos mencionado, que la geografía constituye un factor irrelevante en los procesos de contacto y difusión de fenómenos linguísticos. La Geolinguística, por el contrario, parte ya muy concienciada de estas deficiencias y ofrece una dimensión espacial dinámica en la que son de gran importancia las interacciones espaciales entre las formas lingüísticas:

Instead of giving up on the relationship between linguistic orderliness and geographical dispersion, what is required is a richer set of hypotheses about language variation and a more profound understanding of spatial networks and the diffusion of innovations.

Jack Chambers \& Peter Trudgill (1980: 206)

De este modo, es necesario considerar la posibilidad de correlacionar los datos recogidos por los dialectólogos con predeterminadas unidades geográficas, al igual que los geógrafos han hecho en sus trabajos (Trudgill 1983: 53).

Las relaciones entre la Sociolingiuística y la Geolingiiística, por otra parte, son también evidentes. En lo esencial, no hay diferencias puesto que la segunda forma parte del paradigma global de la primera. Para David Britain (1991), las dos perspectivas se complementan necesariamente:

It is impossible, I will claim, to conceive not only of a geolinguistics without a social content, but also that it is theoretically incorrect to investigate the social phenomena of language devoid of a consideration for the spatial.

David Britain (1991: 11)

La diferencia entre ambas radica en el tratamiento de la espacialidad, dado que, mientras que la Geolingüística se centra en el espacio, la Sociolingüística, la 
laboviana, se centra en la sociedad: «the former asocially quantifies space, whereas ther latter aspatially quantifies society» (David Britain 1991: 209).

Los fundamentos de la Geolingüística son, por tanto, claramente dialectológicos y sociolingüísticos. De hecho, Jack Chambers (1993) la ha llegado a llamar 'dialectología sociolingüística'; igualmente, Peter Trudgill (1983: 51), la ha etiquetado 'dialectología sociolinguísticamente instruida' y ha defendido su entidad siempre desde el rigor y la precisión que requiere la investigación:

I therefore wnat to propose that it may be possible to effect an improvement in our descriptions of geographical dialects - and hence in out ability to explain dialect phenomena - by adopting a geographical and sociolinguistic -influenced methodology [...] If such a methodology proves to be successful, then explanations may follow.

Peter Trudgill (1983: 54)

En cualquier caso, hemos de insistir en la conveniencia de realizar una aproximación a los fenómenos del cambio lingüístico y su difusión desde diversas perspectivas, entre las que no debe faltar nunca la dialectológica, la geolinguística y la sociolinguística. Estas tres aproximaciones son tres tratamientos diferentes, si bien complementarios como Trudgill (1992) defiende, dirigidos hacia un mismo objeto de estudio: los fenómenos de la variación y el cambio linguiístico. Las aproximaciones desarrolladas por los estudios dialectológicos tradicionales y los geolingüísticos se plantean para grupos sociales en un macro-nivel de interacción mientras que las desarrolladas mediante la investigación sociolinguística, o microsociolinguística concretamente, lo hacen para los individuos en un micro-nivel de interacción. Sabemos que, con sus reproducciones cartográficas descriptivas de las diferencias dialectales, los dialectólogos tradicionales han sido considerados en muchas ocasiones como 'meros coleccionistas de mariposas' e incluso practicantes de una especie de 'arqueología lingüística', sobre todo por el desfase existente en los presupuestos teóricos, objetos de estudio, métodos y técnicas empleadas; pero también es cierto que, como Peter Trudgill (1992: 72) sostiene, sin sus descripciones llevadas a cabo entonces antes de que los dialectos regionales tradicionales desaparecieran, no se podría haber hecho uso de esos datos en análisis posteriores como, por ejemplo, los estudios sociolingüísticos de William Labov (1966) en Martha's Vineyard y Nueva York y Trudgill (1974a) en Norwich. No es menos cierto tampoco que los modelos de difusión ofrecidos por la Geolingüística, presentan ventajas, desventajas e incluso inconvenientes. Pero no cabe duda, y en esto coincidimos plenamente con Marinel Gerritsen (1988: 1589), de que, independientemente de su valor probatorio y explicativo, la aproximación a los procesos de difusión lingüística realizada con modelos adaptados de la Geografía Humana es más sólida que la de los dialectólogos. Obviamente, este hecho tiene un 
precio, como son sus inconvenientes: i) la necesidad de recoger y manejar datos poco familiares para los lingüistas, como son las poblaciones, las distancias, los tipos de comunicaciones, los tipos de conexiones (aéreas, terrestres, marítimas), los aspectos físicos y del relieve, etc.; y ii) la división del terreno en áreas uniformes; además de iii) la consecución de datos tanto lingúísticos como sociales de cada una de las celdas para desarrollar el seguimiento del fenómeno en cuestión.

Pero, aunque los modelos de gravedad geolingüísticos consiguen explicar con un elevado grado de precisión el proceso de difusión en un macronivel de interacción, no consiguen, sin embargo, revelarnos nada sobre la interacción real cara-a-cara entre los hablantes de los centros urbanos implicados ni sobre los motivos que les condujeron a adoptar o rechazar la innovación. Las redes sociales de James Milroy \& Lesley Milroy (1985) y el grado de adhesión a éstas (su naturaleza nuclear o periférica) afectan considerablemente a la estructura del habla de una persona y, consiguientemente, a la posibilidad de adopción, y posterior difusión, o rechazo de una innovación. Son los vínculos sociales más débiles de la red social ('weak social ties'), y no los más fuertes ('strong social ties'), los que provocan la trasmisión de las innovaciones lingüísticas en proceso de cambio por requerir un menor esfuerzo, por afectar a más individuos, por tender a escapar de las normas vernáculas y por estar más expuestos a las presiones externas que favorecen el cambio, como puede ser el grado de contacto con hablantes de otras variedades dialectales diferentes. Así, la movilidad regular conduce inevitablemente al debilitamiento de los vínculos con las comunidades locales, tendiendo a actuar como potenciales innovadores aquellos hablantes cuyos contactos sociales y espaciales son heterogéneos. Igualmente, la acomodación lingüística de Howard Giles (1973) a rasgosi lingüísticos prominentes de otros acentos o dialectos dentro del proceso de interacción comunicativa cara-a-cara es crucial para que pueda tener lugar la difusión geográfica de las innovaciones lingüísticas; de hecho, según Trudgill (1986: 40), «diffusion can be said to have taken place, presumably, on the first occasion when a speaker employs a new feature in the absence of speakers of the variety originally containing this feature». También es necesario, pues, una aproximación a estos fenómenos desde el nivel microsociolingüístico de interacción, dado que hay factores actitudinales que pueden estimular o frenar la adopción de las innovaciones y su posterior trasmisión dentro del mismo proceso general. El carácter arbitrario y subjetivo de las actitudes sociales frente al prestigio de las variedades lingüísticas es muy frecuentemente el causante de cambios lingüísticos.

No obstante, hay que ser conscientes de y reconocer que el cambio lingüístico constituye uno de los grandes misterios de la ciencia lingüística, $y$, consiguientemente, un reto para muchas generaciones de lingüistas y filólogos. Creemos, en la línea que Keith Walters (1988), Peter Trudgill (1992) y Walt 
Wolfram \& Natalie Schilling-Estes (en prensa) propugnan, y esto ya hemos defendido en otras ocasiones (Hernández-Campoy 1993a y 1993b), que la existencia de estas diferentes líneas de trabajo -con independencia de lo sofisticadas que sean- provocarán considerables avances en el futuro, además de evidenciar la constante y creciente actividad y solidez de los estudios de la variación.

\section{Conclusión}

Así, una vez que se ha situado el espacio en el lugar que le corresponde incorporando, como hace David Britain (1991), lo geo- en la sociolingüística, tomarán un mayor protagonismo tanto teórico como metodológico los conceptos de espacio y espacialidad en el estudio de la transmisión y mantenimiento de las formas lingüísticas, junto con las dimensiones social y contextual. Además de insistir en la organización espacial de la sociedad (clases), se hace necesario tener en cuenta aquellos aspectos relativos a la organización social del espacio. $\mathrm{Si}$, como defiende Edward Soja (1989: 12), la sociedad es el resultado de la confluencia del tiempo, el espacio y el ser humano, es de desear, pues, que la espacialidad, en tanto que dimensión integrada por los espacios euclidianos, sociales y perceptibles, juegue un papel más relevante en el progreso que hace la investigación sociolingüística en la evidenciación de las interrelaciones existentes entre el lenguaje y la sociedad.

La Sociolingüistica tiende inevitablemente a la integración tanto teórica como metodológica, a pesar de la pluralidad y la diversidad actualmente existente, y de la contraria opinión de Joshua Fishman (1991: 132): «After three decades, sociolinguistics has remained just as it was: a province of linguistics and anthropology, and a rather provincial province at that». Así ha quedado patente la tendencia integradora con los intentos de los sociolingüistas por adaptarse a aquellos aspectos $\mathrm{y}$ artefactos del comportamiento humano que inciden en la comunicación como son la clase social, género, edad, raza, etnia, redes sociales, grupos sociales, actitudes, identidad, política, ideología, etc. De hecho, la Sociolingüística surgió sin base teórica alguna, salvo el postulado de la naturaleza social del lenguaje, $y$ ha ido construyendo toda su fundamentación, afortunadamente creemos, gracias a interdisciplinariedad que ha practicado. $Y$ es más, consideramos que, a la hora de ponernos de acuerdo en el espectro de lenguaje y sociedad, convendría redefinir tanto la ideología como el metalenguaje de los estudios lingüísticos en términos sociolingưísticos, o, de modo más ambicioso, en un espacio multidimensional de la esfera del lenguaje y la comunicación social. Esta integración, basada en la complementariedad paradigmática, permitiría, pensamos, un mayor refinamiento y una mayor precisión de los análisis y un paso 
más adelante en la comprensión del cambio lingüístico y su difusión, contribuyendo, por tanto, a la localización del lenguaje dentro y a través del espacio social, temporal $\mathrm{y}$, por supuesto, geográfico, y constituyendo puntos de convergencia en el perfeccionamiento de la teoría lingüística y, en último término, de nuestro conocimiento de su naturaleza en tanto que capacidad humana.

\section{Referencias Bibliográficas:}

Afendras, E. (1969): «Sociolinguistic History, Sociolinguistic Geography and Bilingualism», en Giornale Internazionali di Sociolinguistica, Roma: Istituto Luigi Sturzo.

Afendras, E. (1970a): «Spatial and Social Aspects of Multilingual Communication», en Pensiero e Linguaggio in Operazioni, vol. 1, 1970, págs. 131-139.

Afendras, E. (1970b): Diffusion Processes in Language: Prediction and Planning, Quebec: Centre International de Recherches sur le Bilinguisme. Mimeo.

Ambrose, J. E. \& Williams, C. H. (1991): «Language Made Visible: Representation in Geolinguistics», en C.H. Williams (ed) (1991), págs. 298-314.

Ammon, U., Dittmar, N. \& Mattherier, K. J. (eds) (1988): Sociolinguistics: An International Handbook of the Science of Language and Society (vol 2), Berlin:

Walter de Gruyter.

Andersen, H. (1986): «Center and Periphery: Adoption, Diffusion and Spread», comunicación presentada en la Conference on Historical Dialectology, 1986, Poznan, Polonia. Posteriormente publicado en J. Fisiak (ed) (1988).

Anderson, C. A. \& Bowman, M. J. (eds) (1965): Education and Economic Development, Chicago: Aldine.

Bailey, C. J. (1973): Variation and Linguistic Theory, Arlington: Center for Applied Linguistics.

Bailey, G., Wikle, T., Tillery, J. \& Sand, L. (1991): «The Apparent Time Construct», en Language Variation and Change, vol. 3, págs. 241-264.

Bailey, G., Wikle, T., Tillery, J., \& Sand, L. (1993): «Some Patterns of Linguistic

Diffusion», en Language Variation and Change, vol. 5, págs. 359-390.

Board, C., Chorley, R., Hagget, P. \& Stoddart, D. R. (eds) (1975): Progress in Geography: International Reviews in Current Research 7, London: Edward Arnold.

Bolton, K. \& Kwok, H. (eds) (1992): Sociolinguistics Today: International Perspectives, London: RKP.

Britain, D. (1991): Dialect and Space: A Geolinguistic Analisis of Speech Variables in the Fens, Colchester: University of Essex (Tesis Doctoral sin publicar). 
Bunge, W. (1962): Theoretical Geography (Lund Studies in Geography, 1), Lund: The Royal University of Lund.

Callary, R. E. (1975): «Phonological Change and the Development of an Urban

Dialect in Illinois», en Language in Society, vol. 4, 1975, págs. 155-169.

Capel Sáez, H. (1981): Filosofía y Ciencia en la Geografia Contemporánea: una Introducción a la Geografía,Barcelona: Barcanova.

Chambers, J. K. (1982): «Geolinguistics of a Variable Rule», en Discussion Papers in Geolinguistics, vol. 5, 1982, págs. 1-17.

Chambers, J. K. (1993): «Sociolinguistic Dialectology», en D. R. Preston (ed), (1993).

Chambers, J. T. \& Trudgill, P. J. (1980): Dialectology, Cambridge: C.U.P.

Chambers, J. K. \& Hardwick, M. (1986): «Comparative Sociolinguistics of a Sound Change in Canadian English», en English World-Wide, vol. 7, 1986, págs. 21-46.

Cooper, R. L. (ed) (1982): Language Spread: Studies in Diffusion and Social Change, Bloomington: Indiana University Press and Center for Applied Linguistics (Washington, DC).

Cooper, R. L. (ed) (1989): Language Planning and Social Change, Cambridge: CUP.

Cooper, R. L. \& Spolsky, B. (eds) (1991): The Influence of Language on Culture and Thought: Essays in Honor of Joshua A. Fishman's Sixty-fifth Birthday, Berlin: Mouton De Gruyter.

Dilthey, W. (1883): Introducción a las Ciencias del Espíritu. Ensayo de una Fundamentación del Estudio de la Sociedad y de la Historia, Madrid: Revista de Occidente; (traducción española de 1956 por Julían Marías y prólogo de José Ortega y Gasset; reeditada en 1980 por Alianza Editorial).

Eckert, P. (1988): «Adolescent Social Structure and the Spread of Linguistic Change», en Language in Society, vol. 17, 1988, págs. 183-207.

Elert, C. (1976): «Gränsen för det syndsvenska bakre $r »$, en Svenska Landsmål och Svenskt Folkliv, págs. 7-20.

Ennen, E. \& Wiegelmann, G. (eds) (1972): Festschrift Matthias Zender, Studien zur Volkskultur, Sprache und Landesgeschichte. Bonn.

Fishman, J. A. (1991): «Putting the 'Socio' Back into the Sociolinguistic Enterprise» (Special article), en International Journal of the Sociology of Language, Vol. 92, 1991, págs. 127-138.

Fisiak, J. (ed) (1988): Historical Dialectology: Regional and Social (Trends in Linguistics, Studies and Monographs No. 37), Berlin: Mouton de Gruyter.

Foldvik, A. K. (1979): «Endring av uttale og spredning av ny uttale: generasjonsskilnader i Brunlanes, Vestfold», en J. Kleiven (ed) (1979).

Foucault, M. (1980): «Questions on Geography», en C. Gordon (ed) (1980). 
Garrison W. L. \& Marble, D. F. (eds) (1967): Quantitative Geography, pt 1. Evanston, Illinois: Northwestern University Department of Geography.

Gass, S., Madden, C., Preston, D., \& Selinker, L. (eds) (1989): Variation in Second Language Acquisition: Psychological Issues, Clevedon: Multilingual Matters.

Gerritsen, M. (1988): «Sociolinguistic Developments as a Diffusion Process», en Ammon, Dittmar \& Matheier (eds) (1988), págs. 1574-1591.

Gerritsen, M. \& Jansen, F. (1980): «The Interplay of Dialectology and Historical Linguistics: Some Refinements of Trudgill's Formula», en P. Maher (ed) (1980), págs. 11-38.

Giddens, A. (1984): The Constitution of Society: Outline of the Theory of Structuralism, Cambridge: Polity Press.

Giles, H. (1973): «Accent mobility: A Model and Some Data», en Anthropological Linguistics, vol. 15, págs. 87-105.

Gordon, C. (ed) (1980): Power/Knowledge: Selected Interviews and Other Writings 1972-77, New York: Pantheon.

Gregory, D. \& Urry, J. (eds) (1985): Social Relations and Spatial Structures, London: MacMillan.

Gregory, J. (1975): Statistical Methods and the Geographer, Longman: London.

Gunnemark, E. V. (1991): Countries, Peoples, and their Languages: the Geolinguistic Handbook, Gothenburg, Sweden.

Hägerstrand, T. (1952): «The Propagation of Innovation Waves», en Lund Studies in Geography, Series B: Human Geography 4. Lund: Gleerup.

Hägerstrand, T. (1957): «Migration and Area: Survey of a Sample of Swedish Migration Fields and Hypothetical Considerations on their Genesis», en Lund Studies in Geography, vol. 13B, 1957, págs. 27-158.

Hägerstrand, T. (1965a): «A Monte Carlo Approach to Diffusion», en Archives Européenes de Sociologie, vol. 6, 1965, págs. 43-67.

Hägerstrand, T. (1965b): «Quantitative Techniques for Analysis of the Spread of Information and Technology», en C.A. Anderson \& M.J. Bowman (eds) (1965).

Hägerstrand, T. (1966): «Aspects of the Spatial Structure of Social Communication and the Diffusion of Information», en Papers of the Regional Science Association, vol. 16, 1966, págs. 27-42.

Hägerstrand, T. (1967a): Innovation Diffusion as a Spatial Process, Chicago: The University of Chicago Press.

Hägerstrand, T. (1967b): «On the Monte Carlo Simulation of Diffusion», en W.L. Garrison \& D.F. Marble (eds) (1967).

Hägerstrand, T. (1969): Diffusion of Innovations, Chicago: University of Chicago Press (traducción al inglés realizada por Allan Pred). 
Hägerstrand, T. (1970): «What about People in Regional Science?», en Papers of the Regional Science Association, vol. 24, 1970, págs. 7-21.

Hagget, P. (1965): Locational Analysis in Human Geography, London: Edward Arnold.

Hard, G. (1972): «Ein geographisches Simulations-modell für die rheinische Sprachgeschichte», en E. Ennen \& G. Wiegelmann (eds)(1972), págs. 25-59.

Harvey, D. (1969): Explanations in Geograph, London: Edward Arnold.

Hattori, S. \& Inoue, K. (eds)(1983): Proceedings of the Thirteenth International Congress of Linguistics, Tokyo: Proceedings Publishing Committee.

Hernández Campoy, J. M. (1993a): «Dialectología Tradicional, Sociolingüística Laboviana y Geolingüística Trudgilliana: Tres Aproximaciones al Estudio de la Variación», en Estudios de Lingüística, vol. 9, 1993.

Hernández Campoy, J. M. (1993b): «Innovating and Conservative Accents in British English: A Brief Quantitative Analysis», en Koiné, vol. III, 1993, págs. 73-91.

Hernández Campoy, J. M. (1993c): Sociolingüistica Británica: Introducción a la Obra de Peter Trudgill, Barcelona: Editorial Octaedro.

Hernández Campoy, J. M. (1996): Modelos de Difusión Geográfica de las Innovaciones Sociolingüisticas en los Acentos del Reino Unido, Murcia: Universidad de Murcia (Tesis Doctoral sin publicar).

Joseph, B. D. \& Janda, R. D. (eds) (en prensa): Handbook of Historical Linguistics, Oxford: Basil Blackwell.

Kerswill, P. (1991): «Babel in Buckinghamshire? Pre-school Children Adquiring Accent Features in the New Town of Milton Keynes», comunicacion presentada en la Conference on Nonstandard Varieties of Language, Estocolmo, Abril de 1991.

Kerswill, P. (1994): A New Dialect in a New City: Children's and Adults' Speech in Milton Keynes. Informe para el Economic and Social Research Council (ESRC), Rf. R000232376, London: ESRC.

Kerswill, P. (1995a): «Children, Adolescents and Language Change», en Working Papers in Linguistics, vol. 2, 1995, University of Reading.

Kerswill, P. (1995b): «Milton Keynes: at the Cutting Edge of Dialect Levelling?», conferencia dada en el ciclo Essex Linguistic Seminar del Department of Language and Linguistics de la Universdad de Essex, 23 de noviembre de 1995.

Kleiven, J. (ed)(1979): Språk of Samfunn: bidrag til en norsk sosiolingvistikk, Oslo: Pax.

Labov, W. (1966): The Social Stratification of English in New York City, Washington, D.C.: Center for Applied Linguistics. 
Larmouth, D. (1981): «Gravity Models, Wave Theory, and Low-structure Regions», en H. Warkentyne (ed) (1981), págs.199-219.

Lippi-Green, R. L. (1989): «Social Network Integration and Language Change in Progress in a Rural Alpine Village», en Language in Society, vol. 18, 1989, págs. 213-234.

Mackey, W. F. (1983): «Sociolinguistics: the Past Decade», en S. Hattori \& K. Inoue (eds)(1983).

Mackey, W. F. (1988): «Geolinguistics: Its Scope and Principles», en C.H. Williams (ed) (1988), págs. 20-46.

Maher, P. J. (ed) (1980): Proceedings of the 3rd International Congress of Historical Linguistics, Amsterdam: Benjamins.

Milroy, J. \& Milroy, L. (1985): «Linguistic Change, Social Network and Speaker Innovation», en Journal of Linguistics, vol. 21, págs. 339-384.

Milroy, L. (1980): Language and Social Networks (Language in Society Series), Oxford: Blackwell (2nd. Edition, 1987).

Newman, S. (1974): «Linguistic Retention and Diffusion in Bella Coola», en Language in Society, vol. 3, 1974, págs. 201-214.

Newmeyer, F. J. (ed) (1988): Linguistics: The Cambridge Survey (4 vols), Cambridge: C.U.P.

Olsson, I. (ed)(1988): MINS 28, Stockholm: Institutionen for Nordiska Sprak, Stokholms Universitet.

Polomé, E. (ed) (1990): Research Guide on Language Change (Trends in Linguistics, Studies and Monographs, 48), New York: Mouton de Gruyter.

Pred, A. (1967): «Behavior and Location», en Lund Studies in Geography, Series B, 1967, Gleerup, Lund.

Pred, A. (1977): «The Choreography of Existence: Comments on Hägerstrand's Time-Geography and its Usefulness», en Economic Geography, vol. 53, 1977, págs. 207-221.

Pred, A. (1985): «The Social Becomes the Spatial, the Spatial Becomes the Social: Enclosures, Social Change and the Becoming of Places in the Swedish Province of Skane», en D. Gregory \& J. Urry (eds) (1985).

Preston, D. (ed) (1993): American Dialect Research, Philadelphia, PA: John Benjamins.

Sandred, K. I. (1988): «From Dialectology to Geolinguistics: A Historical Overview of Some Ways of Dealing with Linguistic Variation», en I. Olsson (ed) (1988), págs. 285-295.

Sayer, A. (1985): «The Difference that Space Makes», en D. Gregory \& J. Urry (eds) (1985).

Schaefer, F. K. (1953): «Exceptionalism in Geography», en Annals of the Asociation of American Geographers, vol. 43, 1953, págs. 226-249. 
Soja, E. (1989): Postmodern Geographies: the Reassertion of Space in Critical Social Theory, London: Verso.

Stewart, J. Q. (1947): «Empirical Mathematical Rules Concerning the Distribution and Equilibrium of Population», en Geographical Review, vol. 37, 1947, págs. 461-485.

Stewart, J. Q. \& Warntz, W. (1958a): «Physics of Population Distribution», en Journal of Regional Science, vol. 1, 1958, págs. 99-123.

Stewart, J.Q. \& Warntz, W. (1958b): «Macrogeography and Social Science», en Geographical Review, vol. 48:2, 1958, págs. 167-184.

Stölting-Richert, W. (1988): «Migration und Sprache», en U. Ammon, N. Dittmar \& K. J. Mattheier (eds) (1988), págs. 1564-1574.

Tollefson, J. W. (1991): Planning Language, Planning Inequality: Language Policy in the Communit, London: Longman.

Trudgill, P. J. (1974a): The Social Differentiation of English in Norwich, Cambridge: C.U.P.

Trudgill, P. J. (1974b): «Linguistic Change and Difussion: Description and Explanation in Sociolinguistic Dialect Geography», en Language in Society, vol. 3, 1974, págs. 215-246. Una versión revisada aparece en P. J. Trudgill (1983), págs. 52-87.

Trudgill, P. J. (1975b): «Linguistic Geography and Geographical Linguistics», en C. Board, R. Chorley, P. Hagget \& D. Stoddart (eds) (1975), págs. 227-252.

Trudgill, P. J. (ed) (1978): Sociolinguistic Patterns in British English, London: Edward Arnold.

Trudgill, P. J. (1983): On Dialect: Social and Geographical Perspectives. Oxford: Blackwell.

Trudgill, P. J. (1986): Dialects in Contact, Oxford: Basil Blackwell.

Trudgill, P. J. (1989): «Interlanguage, Interdialect and Typological Change», en S. Gass, C. Madden, D. Preston \& L. Selinker (eds) (1989).

Trudgill, P. J. (1990): «Dialect Geography», en E. Polomé (ed) (1990), págs. 257271.

Trudgill, P. J. (1990): «Dialect Contact and the Transmission of Linguistic Forms», en W. Wang (ed) (1990).

Trudgill, P. J. (1992): «Dialect Contact, Dialectology and Sociolinguistics», en K. Bolton \& H. Kwok (eds) (1992).

Trudgill, P. J. \& Foxcroft, T. (1978): «On the Sociolinguistics of Vocalic Mergers: Transfer and Approximation in East Anglia», en P. J. Trudgill (ed) (1978), págs. 69-79. También en Trudgill (1983), págs. 88-101.

Urry, J. (1985): «Social Relations, Space and Time», en D. Gregory \& J. Urry (eds) (1985). 
Walters, K. (1988): «Dialectology», en F. J. Newmeyer (ed) (1988), vol. IV, págs. $119-139$.

Wang, W. (ed) (1990): Language Transmission and Change, Oxford: Blackwell.

Warkentyne, H. (ed) (1981): Methods IV: Papers from the 4th International Conference on Methods in Dialectolog, Victoria, British Columbia: University of Victoria.

Williams, C. H. (ed) (1988): Language in Geographic Context, Clevedon, Philadelphia: Multilingual Matters.

Williams, C. H. (ed) (1991): Linguistic Minorities, Society and Territory, Clevedon, Philadelphia: Multilingual Matters.

Williams, C. H. (1994): Called unto Liberty! On Language and Nationalism, Clevedon, Philadelphia: Multilingual Matters.

Wolfram, W. \& Schillin-Estes, N. (en prensa): «Dialectology and Linguistic Diffusion», en B.D. Joseph \& R.D. Janda (eds) (en prensa), versión galerada. 